\title{
East Texas bleeding disorder
}

\author{
INSERM
}

\section{Source}

INSERM. (1999). Orphanet: an online rare disease and orphan drug data base. East Texas bleeding disorder. ORPHA:391320

East Texas bleeding disorder is a rare, genetic, coagulation disorder characterized by easy bruising (without hemarthrosis or spontaneous hematomas), epistaxis, menorrhagia, and excessive bleeding after minor trauma and surgical procedures. Patients present a prolonged prothrombin time and/or activated partial thromboplastin time, normal levels of all coagulation factors, and normal protein C activity. 